\title{
Optimal receiver placement in staring cooperative radar networks for detection of drones
}

\author{
Benjamin Griffin*, Alessio Balleri*, Chris Baker ${ }^{\dagger}$ and Mohammed Jahangir ${ }^{\dagger}$ \\ ${ }^{*}$ Centre for Electronic Warfare, Information and Cyber, Cranfield University, \\ Defence Academy of the United Kingdom, Shrivenham, SN6 8LA \\ ${ }^{\dagger}$ School of Engineering, University of Birmingham \\ Birmingham, B15 2TT, United Kingdom
}

\begin{abstract}
Staring radars use a transmitting static wide-beam antenna and a directive digital array to form multiple simultaneous beams on receive. Because beams are static, the radar can employ long integration times that facilitate the detection of slow low-RCS targets, such as drones, which present a challenge to traditional air surveillance radar. Typical low altitude trajectories employed by drones often result in lowgrazing angle multipath effects which are difficult to mitigate with a monostatic radar alone. The use of multiple spatially separated receivers cooperating with the staring transmitters in a multistatic network allows multi-perspective target acquisitions that can help mitigate multipath and ultimately enhance the detection of drones. This paper investigates how varying the network geometry affects the estimation performance of a targets position and velocity in a multipath free scenario. The optimal geometry is found by minimising the trace of the CramérRao Lower Bound (CRLB) of the Maximum Likelihood (ML) estimates of range and Doppler using the Coordinate Descent (CD) algorithm. The network estimation accuracy performance is verified using Monte Carlo simulations and an ML Estimator on the target parameter estimates.

Index Terms-Radar, Network Optimisation, Estimation, Drones
\end{abstract}

\section{INTRODUCTION}

The robust detection of drones is an ongoing problem in the radar domain. Due to their small size, low flight profile and slow speed, drones present a unique challenge to traditional air surveillance radar. Radar networks have seen a surge of interest as they have the potential to improve the detection of drones [1], [2]. A list of required capabilities for a radar system to be able to detect and track drones has been presented by Poitevin et al [3] and include:

- Sensitivity for very small detectable signature

- Fast refresh rate

- Height measurement capability

- Large volumetric coverage requirements

- Nuisance elimination

- System cost

Staring array radar exist that can detect small drones out to a range of $5 \mathrm{~km}$ and satisfies the sensitivity, fast refresh

The authors thank Thales Aveillant Ltd and Cranfield Defence and Security for funding this work under the Cranfield University 50:50 Industrial PhD scheme. rate and height measurement capability [4]. Measurements from the Aveillant Gamekeeper radar have reported in-flight Doppler spectrogram characterisation of drones and birds, aiding discrimination between two otherwise very similar targets [5].

The additional information gained by the spatial diversity of a network can help mitigate multipath fading and terrain masking due to the drones low flight altitude by observing the target from multiple receivers [6]. A combined network of receivers cooperating with a staring transmitter has the potential to satisfy the desired requirements for a drone detection radar system. Radar networks have been proposed in the past for drone detection in passive radar. Small WiFi-based networks consisting of a single transmitter and two receivers have been studied by Milani et al [7]. These networks use the angle of arrival and time difference of arrival measurements for estimating target position in $2 \mathrm{D}$ and $3 \mathrm{D}$ [8], [9]. While WiFi based methods can be considered a green system and ideal for urban environments, they are limited in range. The use of Digital Terrestrial Multimedia Broadcast (DTMB) signals are better suited as the transmitters are generally mounted high up, and have been shown to detect drones over greater distances [10] [11].

Active radar networks are generally more appropriate than passive networks as the transmitted signal is precisely known, and its parameters can be adjusted to match the requirements of the network. The NetRAD radar is one of the few small scale active radar networks that has been used to look at the signatures of drones [12], [13]. The NetRAD time domain information is coupled with the targets micro-Doppler signature improving the discrimination between ground clutter and the target.

The performance of a radar network depends on many factors such as the environment the network is operating in as well as any radio propagation issues. Performance is also dependent on the geometry of the network (the positions of the transmitters and receivers) relative to the location of the target [14]. In the radar domain, the performance of the network can be measured by the target localisation error. There exists extensive work on target localisation using time 
delay, angle of arrival and Doppler shift measurements [15], [16]. The optimisation of the geometry of sensor networks using the measurements of the targets Doppler shift to localise the target has been studied for fixed sensors in [17], [18], and for mobile sensors [19], [20]. The above considers sensors which act as both the transmitter and receivers, and does not exploit the bistatic signals from the other sensors. Convex optimisation has be used for sensor placement in MIMO networks over a discrete set of possible positions, methods to reduce computation time are discussed in [21]. An analysis of the number of receivers needed in a multistatic network to achieve the desired localisation error of target using range measurements is given by [22]. The aforementioned demonstrates that the performance of such a multistatic radar network is mainly determined by the number of bistatic pairs.

Both the passive and active radar networks mentioned above suffer from a limited coverage area. The combined network of receivers cooperating with a staring transmitter has the potential to resolve this and enhance the detection of drones. In this paper, the trace of the CRLB of the target parameter estimator is used to measure the performance of the radar network. Minimising the trace of the CRLB is often referred to as the A-optimality criterion, and represents minimising the sum of the variances of the estimated parameters. The measurement model uses both range and Doppler measurements from each of the receivers to estimate the target parameters.

\section{THEORETICAL MODEL}

The aim of this paper is to determine the radar network geometry that estimates the targets position and velocity with the smallest possible error. The vector $\boldsymbol{\theta}=\left(\begin{array}{llll}p_{x} & p_{y} & v_{x} & v_{y}\end{array}\right)^{T}$ is the target parameter vector, where $\boldsymbol{p}=\left(\begin{array}{ll}p_{x} & p_{y}\end{array}\right)^{T}$ and $\boldsymbol{v}=\left(\begin{array}{ll}v_{x} & v_{y}\end{array}\right)^{T}$ represent the position and velocity of the target in the 2D Cartesian plane. Consider a 2D multistatic radar network consisting of a single static staring transmitter and $N$ omnidirectional receivers. Denote the position of the transmitter as $\boldsymbol{p}^{(T)}$ and the position of the nth receiver as $\boldsymbol{p}_{n}^{(R)}$.

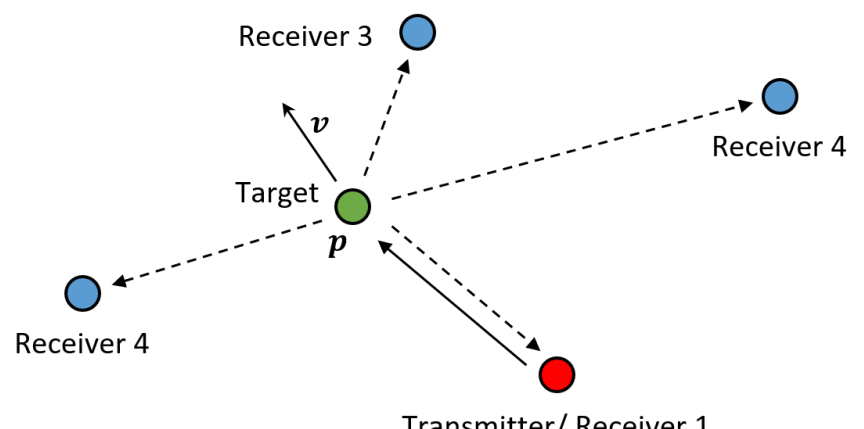

Transmitter/ Receiver 1

Fig. 1. Example Network Geometry

Fig. 1 illustrates a multistatic radar network consisting of a single transmitter/ receiver, three receivers and a single target. The signal received at each receiver will be different and depends on the geometry of the radar network and the relative position and velocity of the target.

\section{A. Signal Model}

Each receiver in the network receives a noisy, time-delayed and Doppler-shifted version of the transmitted signal with complex envelope $u(t)$ which, after down-conversion, may be expressed as

$$
s_{n}(t)=\sqrt{E_{n}} u\left(t-\tau_{n}\right) e^{2 \pi i f_{n} t}+w_{n}(t)
$$

where $\tau_{n}$ and $f_{n}$ represent the time delay and the Doppler shift received at the nth receiver respectively. The complex envelope $u(t)$ is normalised so $E_{n}$ is the energy of the received signal. The signal noise is a complex zero-mean Gaussian white random process. Estimating the time delay and Doppler shift of the received signal has been shown to be dependent on the ambiguity function of $u(t)$ and the signal to noise ratio at the receiver [23]. The ambiguity function of the transmitted signal determines the CRLB for estimating the time delay and Doppler shift of the signal. It has also been shown that the CRLB can be used as the covariance matrix of the corresponding MLE estimator when the energyto-noise ratio is high [23]. Table I lists the parameters of the Linear Frequency Modulation (LFM) pulse which has been used in this paper. Using the above notation the CRLB for

TABLE I

LFM SIGNAL PARAMETERS

\begin{tabular}{c|c|c}
\hline Parameter & Symbol & Value \\
\hline \hline Bandwidth & $\mathrm{B}$ & $1 \mathrm{MHz}$ \\
\hline Pulse Repetition Interval & PRI & $100 \mu \mathrm{s}$ \\
\hline Number of Pulses & $\mathrm{M}$ & 16 \\
\hline Pulse Length & $T$ & $50 \mu \mathrm{s}$ \\
\hline Wavelength & $\lambda$ & $0.21 \mathrm{~m}$ \\
\hline
\end{tabular}

estimating the time delay and Doppler shift from (1) for a complex envelope consisting of $M$ LFM pulses, is

$$
\begin{aligned}
\sigma_{\tau}^{2} & =\frac{1}{3 d} \pi^{2}\left(T^{2}+\mathrm{PRI}^{2}\left(M^{2}-1\right)\right) \\
\sigma_{f}^{2} & =\frac{1}{3 d} \pi^{2} B^{2} \\
\sigma_{\tau f} & =\frac{1}{3 d} \pi^{2} B T
\end{aligned}
$$

where $d=\frac{1}{9} \pi^{4} B^{2} \mathrm{PRI}^{2}\left(M^{2}-1\right)$ [24], [25]. The CRLB for estimating the range and Doppler shift from (1) can be found by defining $r=c \tau$, where $r$ is the targets range and $c$ is the speed of light. The measurement model is

$$
\begin{aligned}
\hat{r}_{n} & =r_{n}(\boldsymbol{\theta})+e_{r_{n}} \\
\hat{f}_{n} & =f_{n}(\boldsymbol{\theta})+e_{f_{n}} \\
\Sigma_{n} & =\frac{1}{\mathrm{SNR}_{n}}\left(\begin{array}{cc}
\sigma_{r}^{2} & \sigma_{r f} \\
\sigma_{f r} & \sigma_{f}^{2}
\end{array}\right)
\end{aligned}
$$

where $\hat{r}_{n}$ and $\hat{f}_{n}$ are the MLE estimates of the range and Doppler at the nth receiver. The Gaussian measurement error is represented by $e_{r_{n}}$ and $e_{f_{n}}$ with zero-mean and covariance $\Sigma_{n}$. These measurements are dependent on the target parameters $\boldsymbol{\theta}$ and the geometry of the radar network. 


\section{B. Fusion of the Range and Doppler measurements}

Each receiver in the network can output a range and Doppler shift measurement using the above process. The measurements from the radar network are combined into a single vector $\boldsymbol{z}$.

$$
\boldsymbol{z}=\boldsymbol{\mu}(\boldsymbol{\theta})+\boldsymbol{e}=\left(\begin{array}{c}
r_{1}(\boldsymbol{\theta}) \\
f_{1}(\boldsymbol{\theta}) \\
\vdots \\
r_{N}(\boldsymbol{\theta}) \\
f_{N}(\boldsymbol{\theta})
\end{array}\right)+\left(\begin{array}{c}
e_{r_{1}} \\
e_{f_{1}} \\
\vdots \\
e_{r_{N}} \\
e_{f_{N}}
\end{array}\right)
$$

The vector $\boldsymbol{z}$ is a Gaussian random variable with mean value $\boldsymbol{\mu}(\boldsymbol{\theta})$ and covariance matrix

$$
\boldsymbol{\Sigma}=\left(\begin{array}{cccc}
\Sigma_{1} & 0 & \cdots & 0 \\
0 & \Sigma_{2} & \cdots & 0 \\
\vdots & \vdots & \ddots & \vdots \\
0 & 0 & 0 & \Sigma_{N}
\end{array}\right)
$$

The mean value $\boldsymbol{\mu}(\boldsymbol{\theta})$ contains the target range and Doppler shift at all receivers. The vector $e$ represents the measurement error. The covariance matrix $\boldsymbol{\Sigma}$ is a block diagonal matrix because the measurements from each receiver in the network are independent. Each diagonal block contains the range and Doppler shift covariance matrix from each individual receiver in the network. The Fisher Information Matrix (FIM) for a Gaussian random vector is given by [26]

$$
\begin{aligned}
{[I(\boldsymbol{\theta})]_{i j}=} & {\left[\frac{\partial \boldsymbol{\mu}(\boldsymbol{\theta})}{\partial \theta_{i}}\right]^{T} \boldsymbol{\Sigma}^{-1}\left[\frac{\partial \boldsymbol{\mu}(\boldsymbol{\theta})}{\partial \theta_{j}}\right] } \\
& +\frac{1}{2} \operatorname{tr}\left[\boldsymbol{\Sigma}^{-1}(\boldsymbol{\theta}) \frac{\partial \boldsymbol{\Sigma}(\boldsymbol{\theta})}{\partial \theta_{i}} \boldsymbol{\Sigma}^{-1}(\boldsymbol{\theta}) \frac{\partial \boldsymbol{\Sigma}(\boldsymbol{\theta})}{\partial \theta_{j}}\right]
\end{aligned}
$$

The FIM (6) is simplified as the covariance matrix $\boldsymbol{\Sigma}$ is not a function of the targets parameters. As $\boldsymbol{\Sigma}$ is block diagonal matrix (6) can be written as a sum of matrices where each term in the sum depends on the measurements from a single receiver as

$$
\begin{aligned}
{[I(\boldsymbol{\theta})]_{i j} } & =\sum_{n=1}^{N}\left[\frac{\partial \boldsymbol{\mu}_{n}(\boldsymbol{\theta})}{\partial \theta_{i}}\right]^{T} \Sigma_{n}^{-1}\left[\frac{\partial \boldsymbol{\mu}_{n}(\boldsymbol{\theta})}{\partial \theta_{j}}\right] \\
{\left[\frac{\partial \boldsymbol{\mu}_{n}(\boldsymbol{\theta})}{\partial \theta_{i}}\right] } & =\left[\begin{array}{c}
\frac{\partial r_{n}(\boldsymbol{\theta})}{\partial \theta_{i}} \\
\frac{\partial f_{n}(\boldsymbol{\theta})}{\partial \theta_{i}}
\end{array}\right] .
\end{aligned}
$$

\section{Range and Doppler Derivatives}

The FIM (7) depends on the derivatives of the range and Doppler measurements

$$
\begin{aligned}
r_{n}(\boldsymbol{\theta}) & =\left|\boldsymbol{p}^{(T)}-\boldsymbol{p}\right|+\left|\boldsymbol{p}_{n}^{(R)}-\boldsymbol{p}\right| \\
f_{n}(\boldsymbol{\theta}) & =\frac{\boldsymbol{v}}{\lambda} \cdot\left(\frac{\left(\boldsymbol{p}^{(T)}-\boldsymbol{p}\right)}{\left|\boldsymbol{p}^{(T)}-\boldsymbol{p}\right|}+\frac{\left(\boldsymbol{p}_{n}^{(R)}-\boldsymbol{p}\right)}{\left|\boldsymbol{p}_{n}^{(R)}-\boldsymbol{p}\right|}\right)
\end{aligned}
$$

with respect to the target parameters. Define $\beta$ as the angle between the transmitter and the target and $\alpha_{n}$ as the angle between the nth receiver and the target, as shown in Fig. 2 .

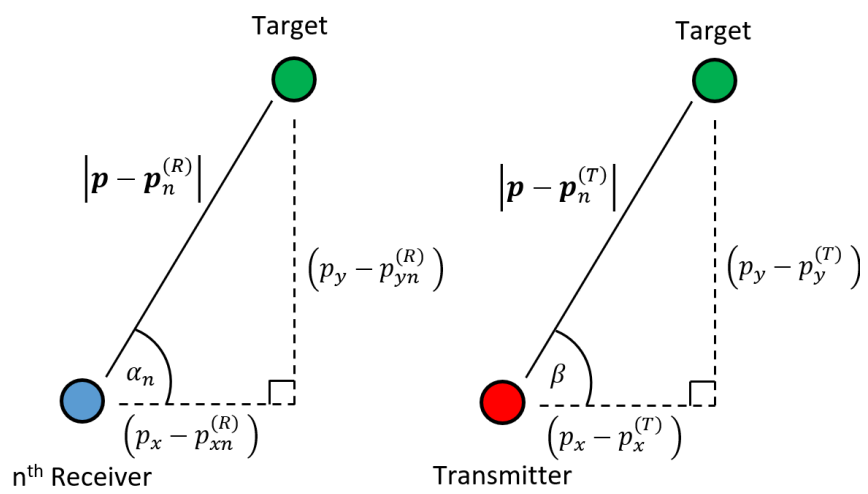

Fig. 2. Receiver and Transmitter Angles

Define $b=\frac{v_{x} \sin (\beta)-v_{y} \cos (\beta)}{\left|\boldsymbol{p}^{(T)}-\boldsymbol{p}\right|}$ as the angular velocity of the target with respect to the transmitter. Similarly, define $a_{n}=$ $\frac{v_{x} \sin \left(\alpha_{n}\right)-v_{y} \cos \left(\alpha_{n}\right)}{\left|\boldsymbol{p}_{n}^{(R)}-\boldsymbol{p}\right|}$ as the angular velocity of the target with respect to the nth receiver. The derivatives of the range and Doppler measurements can be written in terms of the Sines and Cosines of the angles the transmitter and receivers make with the target (9) as

$$
\begin{aligned}
& \frac{\partial r_{n}(\boldsymbol{\theta})}{\partial x}=\cos (\beta)+\cos \left(\alpha_{n}\right) \\
& \frac{\partial r_{n}(\boldsymbol{\theta})}{\partial y}=\sin (\beta)+\sin \left(\alpha_{n}\right) \\
& \frac{\partial r_{n}(\boldsymbol{\theta})}{\partial \dot{x}}=0 \\
& \frac{\partial r_{n}(\boldsymbol{\theta})}{\partial \dot{y}}=0 \\
& \frac{\partial f_{n}(\boldsymbol{\theta})}{\partial x}=-\frac{1}{\lambda}\left(b \sin (\beta)+a_{n} \sin (\alpha)\right) \\
& \frac{\partial f_{n}(\boldsymbol{\theta})}{\partial y}=\frac{1}{\lambda}\left(b \cos (\beta)+a_{n} \cos \left(\alpha_{n}\right)\right) \\
& \frac{\partial f_{n}(\boldsymbol{\theta})}{\partial \dot{x}}=-\frac{1}{\lambda}\left(\cos (\beta)+\sin \left(\alpha_{n}\right)\right) \\
& \frac{\partial f_{n}(\boldsymbol{\theta})}{\partial \dot{y}}=-\frac{1}{\lambda}\left(\sin (b)+\sin \left(a_{n}\right)\right)
\end{aligned}
$$

The resulting FIM is a 4D matrix, the inverse of which is the CRLB for estimating $\boldsymbol{\theta}$. For radar networks consisting of more than two receivers numerical methods are required to determine the CRLB. The optimal geometry is then the one that minimises the trace of the CRLB.

\section{Maximum Likelihood Estimator}

The MLE is an estimator with performance that reaches the theoretical CRLB at high SNR [26]. Define $\Lambda(\boldsymbol{\theta} ; \boldsymbol{z})$ as the likelihood of the parameter $\boldsymbol{\theta}$ taking the values given by 
the targets estimated range and Doppler shift at the receivers, given by $z$. The log-likelihood function can be expressed as

$$
\begin{aligned}
\ln \Lambda(\boldsymbol{\theta} ; \boldsymbol{z})= & -2 N \ln (2 \pi)-\ln |\boldsymbol{\Sigma}| \\
& -\frac{1}{2} \sum_{n=1}^{N}\left(\boldsymbol{z}_{n}-\boldsymbol{\mu}_{n}\right)^{T} \Sigma_{n}^{-1}\left(\boldsymbol{z}_{n}-\boldsymbol{\mu}_{n}\right) .
\end{aligned}
$$

The parameter $\boldsymbol{\mu}_{n}$ contains the noiseless range and Doppler shift measurements.

\section{Simulation Results}

The network needs to estimate four target parameters and as each receiver in the network generates two measurements, at least two receivers are required. To ensure that the FIM is nonsingular for all possible geometries, consider a radar network consisting of a single transmitter and four receivers.

TABLE II

EXAMPLE NETWORK GEOMETRY

\begin{tabular}{c|c|cc}
\hline Label & Position & $\mathrm{x}[\mathrm{m}]$ & $\mathrm{y}[\mathrm{m}]$ \\
\hline Tx 1 & $\boldsymbol{p}^{(T)}$ & 0 & 0 \\
\hline Rx 1 & $\boldsymbol{p}_{1}^{(R)}$ & 0 & 0 \\
\hline Rx 2 & $\boldsymbol{p}_{2}^{(R)}$ & 500 & -1500 \\
\hline Rx 3 & $\boldsymbol{p}_{3}^{(R)}$ & -2000 & 2500 \\
\hline
\end{tabular}

We consider a network arranged around a central monostatic radar (labelled by $\mathrm{Tx} 1$ and $\mathrm{Rx} 1$ ). Two additional fixed receivers ( $\mathrm{Rx} 2$ and $\mathrm{Rx} 3$ ) and one moveable receiver ( $\mathrm{Rx}$ 4) tethered to the monostatic radar Fig3. The positions of the fixed components are given in Table II. The tethered receiver can be placed on a circle of radius $1 \mathrm{~km}$ around the monostatic radar. The geometry of the network is varied by changing the angle of the tethered receiver. The target is located at $\boldsymbol{p}=(2000 \mathrm{~m} 2000 \mathrm{~m})^{T}$ and has velocity $\boldsymbol{v}=\left(\begin{array}{ll}1 \mathrm{~ms}^{-1} & 2 \mathrm{~ms}^{-1}\end{array}\right)^{T}$. The LFM pulse described in Table I is used with transmitted power set such that the received SNR of the monostatic radar is $30 \mathrm{~dB}$.

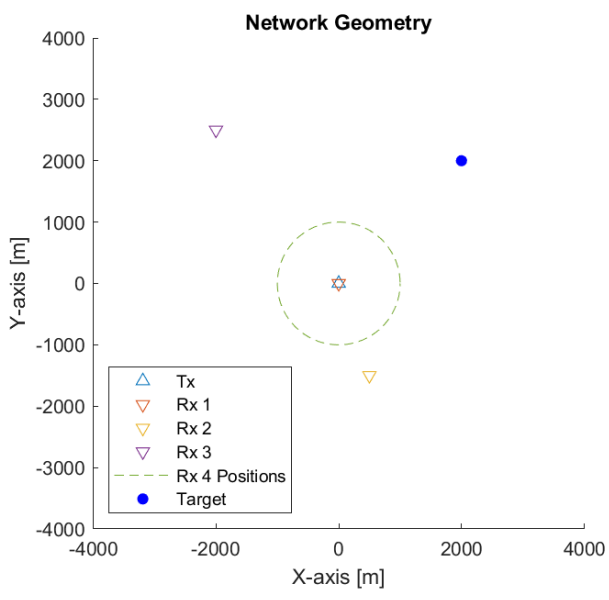

Fig. 3. Example Radar Network Geometry

\section{A. Network Geometry Optimisation}

This model has only one variable, the position of the tethered receiver along a circle of radius $1 \mathrm{~km}$ centred on the monostatic radar. The network is optimised by sweeping the receiver around the monostatic radar and selecting the position which minimises the trace of the CRLB. Fig. 4 shows the trace of the CRLB against the angle of the tethered receiver with respect to the $\mathrm{X}$-axis.

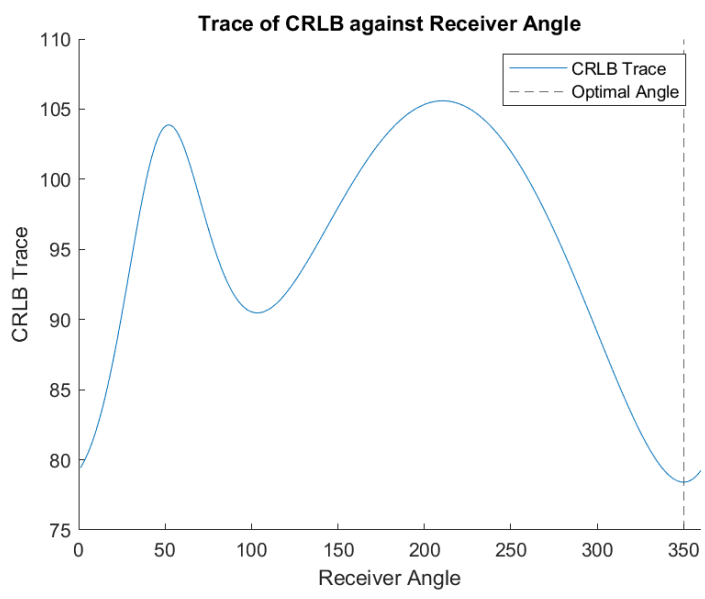

Fig. 4. Trace of CRLB against Receiver Angle

Fig. 4 shows two local minima at $100^{\circ}$ and $350^{\circ}$, the global minimum is at $350^{\circ}$. The best place for the tethered receiver $(\operatorname{Rx} 4)$ is at $\boldsymbol{p}_{4}^{(R)}=\left(\begin{array}{ll}985 \mathrm{~m} & -174 \mathrm{~m}\end{array}\right)^{T}$. Note that the minimum points are symmetric about the transmitter-target line.

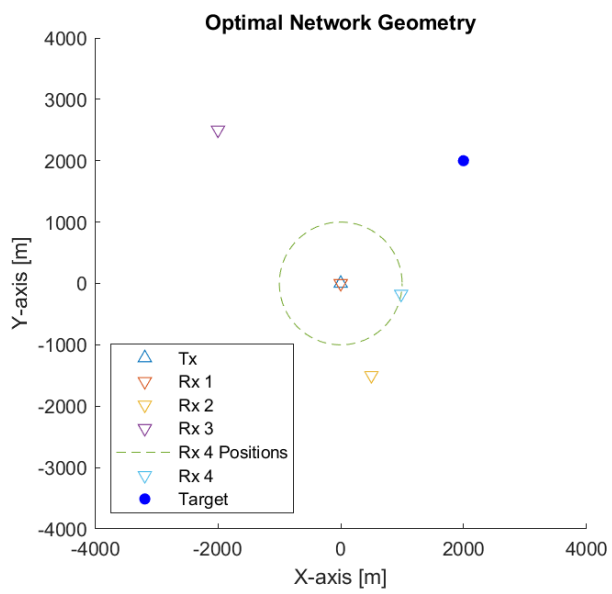

Fig. 5. Optimal Network Geometry

\section{B. MLE Simulation Results}

Monte Carlo simulations of the MLE are used to verify the performance of the derived optimal network geometry. The $\mathrm{CD}$ algorithm is used to determine Monte Carlo samples in the simulation. The $\mathrm{CD}$ algorithm iterates along the parameters 
of coordinates of a multivariate function optimising over one variable at a time [27].

$$
\hat{\boldsymbol{\theta}}=\underset{\boldsymbol{\theta}}{\arg \max } \ln \Lambda(\boldsymbol{\theta} ; \boldsymbol{z})
$$

The CD algorithm starts by randomly selecting initial variable values $\boldsymbol{\theta}^{0}$. The algorithm then selects a single variable and minimises along the direction of the chosen variable, keeping all other variables constant. The algorithm terminates when the sequence $\left(\boldsymbol{\theta}^{k}\right)$ converges. A full description of the CD algorithm is given in Algorithm 1.

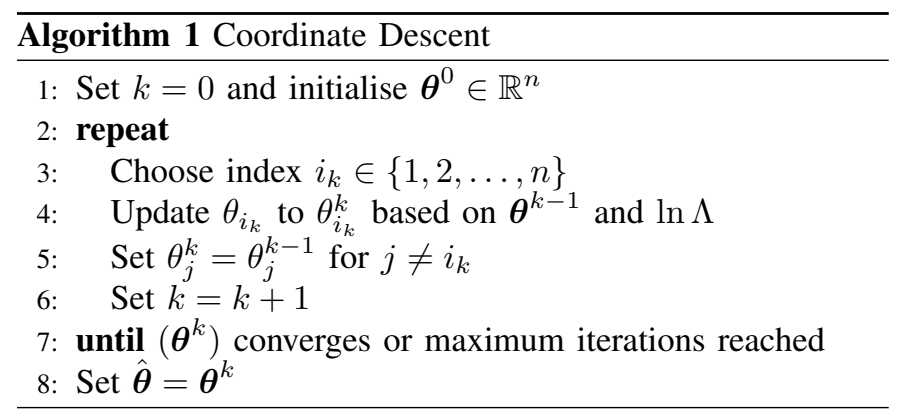

The update step renews the selected coordinate $\theta_{i_{k}}$ by maximising the log-likelihood with respect to $\theta_{i_{k}}$ while keeping the remaining coordinates fixed

$$
\theta_{i_{k}}^{k}=\underset{\theta_{i_{k}}}{\arg \max }\left\{\ln \Lambda\left(\theta_{1}^{k-1}, \ldots, \theta_{i_{k}}, \ldots, \theta_{n}^{k-1} ; \boldsymbol{z}\right)\right\} .
$$

Due to the distributed nature of the network each receiver in the network receives a different amount of energy which depends on their relative position to the target and the transmitter. As a result each receiver has a different SNR. The MLE simulation varies the transmitted power such that the SNR at the monostatic receiver runs from $1 \mathrm{~dB}$ to $30 \mathrm{~dB}$.

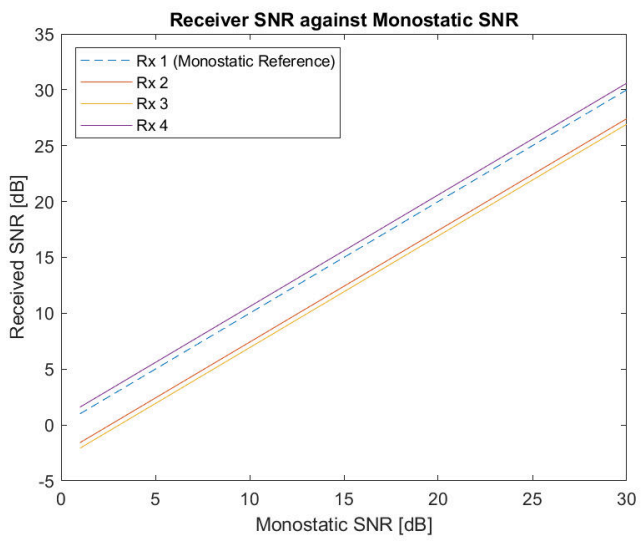

Fig. 6. Receiver SNR against Monostatic Receiver

Fig. 6 shows that two of the receivers ( $\mathrm{Rx} 2$ and $\mathrm{Rx} 3$ ) receive less energy than the monostatic reference while $\mathrm{Rx}$ 4 receives more energy. Fig. 7-10 plots the root mean square error (RMSE) for each of the four target parameters against the monostatic SNR. The SNR varies between $1 \mathrm{~dB}$ and 30 dB. Each data point was calculated using 5000 Monte Carlo trials.

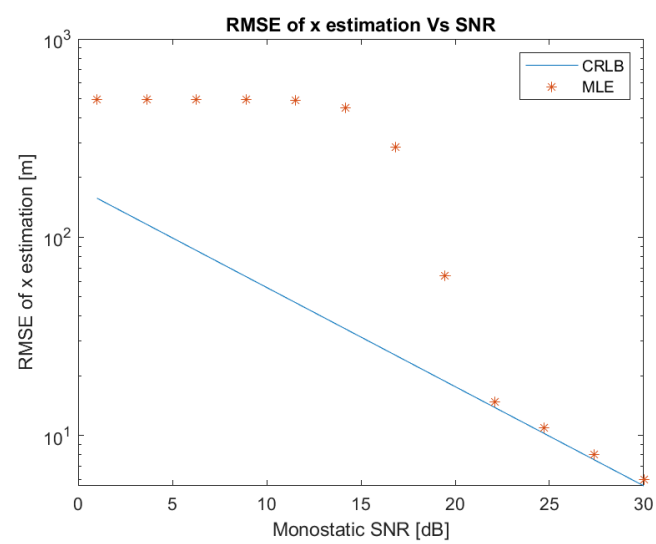

Fig. 7. x RMSE Vs SNR

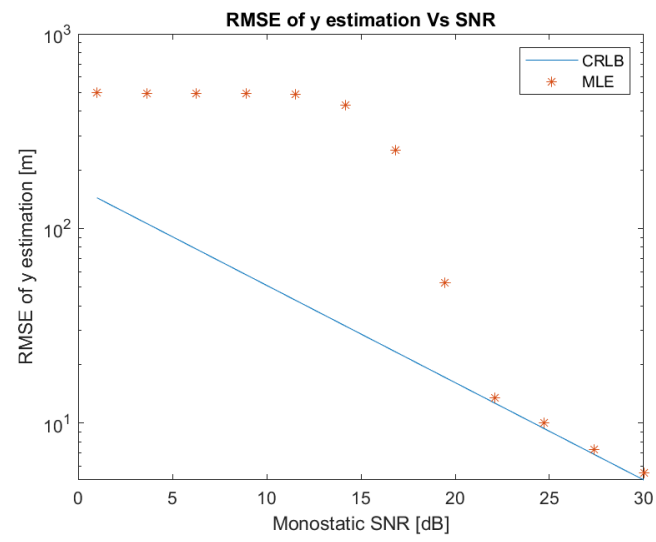

Fig. 8. y RMSE Vs SNR

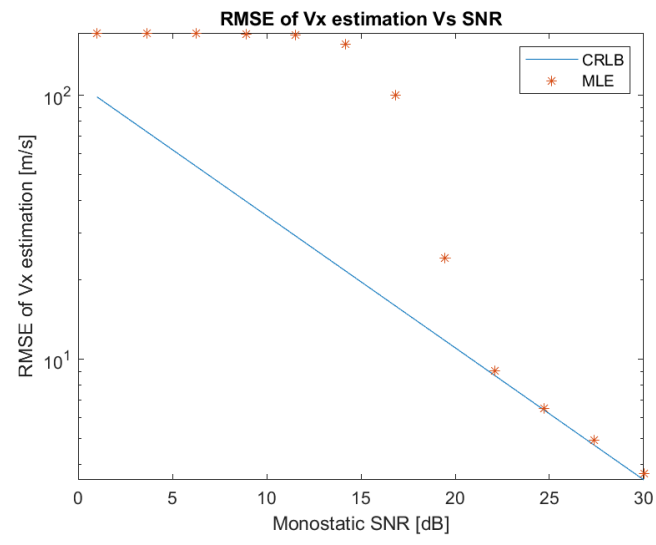

Fig. 9. Vx RMSE Vs SNR 


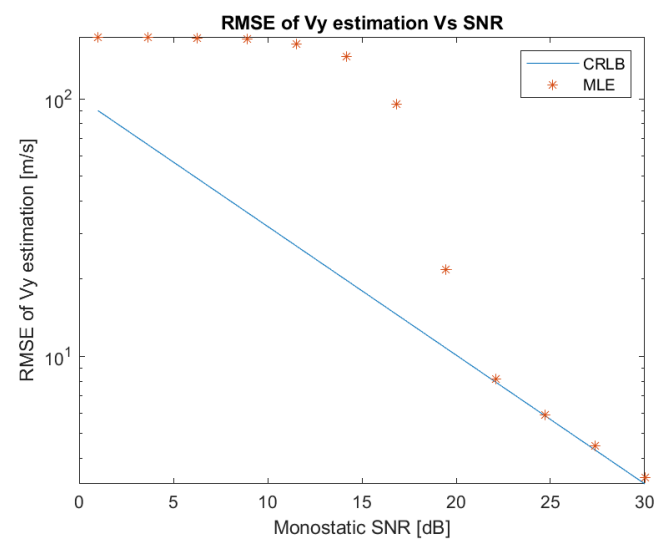

Fig. 10. Vy RMSE Vs SNR

The results of the Monte Carlo simulations show that at low SNR the MLE is unable to distinguish the target signal from the noise. At high SNR $(22 \mathrm{~dB})$ the MLE attains the theoretical CRLB for each of the four target parameters verifying the accuracy of the selected optimal network geometry.

\section{CONCLUSION}

This paper describes a method for placing the receivers in a multistatic radar network such that the estimation error of the targets position and velocity is minimised. The trace of the CRLB is used to measure the performance of the network, and the geometry that minimises the trace is said to be optimal. The performance of the optimal geometry is then verified using Monte-Carlo simulations of the MLE.

A natural extension of the work is to include a third dimension representing the height of the target. The inclusion of the height parameter enables incorporation of the effects of ground multipath reflections on the received signal. The model can be extended to include the angle of arrival measurements from the staring radar. The $\mathrm{CD}$ algorithm can also be used to determine the optimal geometry for networks with multiple moveable transmitters or receivers. The model could be constrained by considering using non-omnidirectional antennas and selecting the optimal geometry from a discrete set of receiver positions.

\section{REFERENCES}

[1] Hugh Griffiths. Developments in bistatic and networked radar. Proceedings of 2011 IEEE CIE International Conference on Radar, RADAR 2011, 1:10-13, 2011.

[2] Hugh Griffiths. Multistatic, MIMO and networked radar: The future of radar sensors? European Microwave Week 2010, EuMW2010: Connecting the World, Conference Proceedings - European Radar Conference, EuRAD 2010, (October):81-84, 2010.

[3] Pierre Poitevin, Michel Pelletier, and Patrick Lamontagne. Challenges in detecting UAS with radar. In 2017 International Carnahan Conference on Security Technology (ICCST), volume 2017-Octob, pages 1-6. IEEE, oct 2017.

[4] Mohammed Jahangir and Chris Baker. Persistence surveillance of difficult to detect micro-drones with L-band 3-D holographic $\operatorname{radar}^{\mathrm{TM}}$. In 2016 CIE International Conference on Radar (RADAR), pages 1-5. IEEE, oct 2016.

[5] Mohammed Jahangir and Chris J. Baker. Extended dwell Doppler characteristics of birds and micro-UAS at l-band. In 2017 18th International Radar Symposium (IRS), pages 1-10. IEEE, jun 2017.
[6] Peter Wellig, Peter Speirs, Christof Schuepbach, Roland Oechslin, Matthias Renker, Urs Boeniger, and Hans Pratisto. Radar Systems and Challenges for C-UAV. In 2018 19th International Radar Symposium (IRS), volume 2018-June, pages 1-8. IEEE, jun 2018.

[7] Ileana Milani, Fabiola Colone, Carlo Bongioanni, and Pierfrancesco Lombardo. WiFi emission-based vs passive radar localization of human targets. In 2018 IEEE Radar Conference (RadarConf18), pages 13111316. IEEE, apr 2018.

[8] Ileana Milani, Fabiola Colone, and Pierfrancesco Lombardo. 2D localization with WiFi passive radar and device-based techniques: An analysis of target measurements accuracy. In Proceedings International Radar Symposium, volume 2018-June, pages 1-10. IEEE, jun 2018.

[9] T. Martelli, F. Murgia, F. Colone, C. Bongioanni, and P. Lombardo. Detection and 3D localization of ultralight aircrafts and drones with a WiFi-based Passive Radar. In International Conference on Radar Systems (Radar 2017), volume 2017. Institution of Engineering and Technology, 2017.

[10] Yuqi Liu, Xianrong Wan, Hui Tang, Jianxin Yi, Yiyao Cheng, and Xun Zhang. Digital television based passive bistatic radar system for drone detection. In 2017 IEEE Radar Conference (RadarConf), pages 14931497. IEEE, may 2017.

[11] Gao Fang, Jianxin Yi, Xianrong Wan, Yuqi Liu, and Hengyu Ke. Experimental Research of Multistatic Passive Radar with a Single Antenna for Drone Detection. IEEE Access, 6:33542-33551, 2018.

[12] Folker Hoffmann, Matthew Ritchie, Francesco Fioranelli, Alexander Charlish, and Hugh Griffiths. Micro-Doppler based detection and tracking of UAVs with multistatic radar. 2016 IEEE Radar Conference, RadarConf 2016, pages 1-6, 2016.

[13] Matthew Ritchie, Francesco Fioranelli, Hervé Borrion, and Hugh Griffiths. Multistatic micro-Doppler radar feature extraction for classification of unloaded/loaded micro-drones. IET Radar, Sonar \& Navigation, 11(1):116-124, jan 2017

[14] E. Hanle. Survey of bistatic and multistatic radar. IEE Proceedings $F$ Communications, Radar and Signal Processing, 133(7):587, 1986.

[15] Kutluyıl Doğançay and Hatem Hmam. Optimal angular sensor separation for AOA localization. Signal Processing, 88(5):1248-1260, may 2008.

[16] Seyed Amir Reza Kazemi, Rouhollah Amiri, and Fereidoon Behnia. Efficient Convex Solution for 3-D Localization in MIMO Radars Using Delay and Angle Measurements. IEEE Communications Letters, 23(12):2219-2223, dec 2019.

[17] Iman Shames, Adrian N. Bishop, Matthew Smith, and Brian D. O. Anderson. Doppler Shift Target Localization. IEEE Transactions on Aerospace and Electronic Systems, 49(1):266-276, jan 2013.

[18] Shiyu Zhao, Ben M. Chen, and Tong H. Lee. Optimal sensor placement for target localisation and tracking in 2D and 3D. International Journal of Control, 86(10):1687-1704, 2013.

[19] Ngoc Hung Nguyen and Kutluyil Dogancay. Optimal sensor placement for Doppler shift target localization. In 2015 IEEE Radar Conference (RadarCon), volume 2015-June, pages 1677-1682. IEEE, may 2015.

[20] Sheng Xu and Kutluyil Dogancay. Optimal sensor deployment for 3D AOA target localization. In 2015 IEEE International Conference on Acoustics, Speech and Signal Processing (ICASSP), volume 2015Augus, pages 2544-2548. IEEE, apr 2015.

[21] Jianxin Yi, Xianrong Wan, Henry Leung, and Min Lu. Joint Placement of Transmitters and Receivers for Distributed MIMO Radars. IEEE Transactions on Aerospace and Electronic Systems, 53(1):122-134, feb 2017.

[22] I. M. Ivashko, O. A. Krasnov, and A. G. Yarovoy. Some aspects of the multistatic radar network topology optimization. In Proceedings International Radar Symposium, volume 2016-June, 2016.

[23] H L Van Trees. Detection, estimation, and modulation theory, Part 3 - Radar-sonar signal processing and Gaussian signals in noise, volume 18. John Wiley, 1972.

[24] Alessio Balleri and Alfonso Farina. Ambiguity function and accuracy of the hyperbolic chirp: Comparison with the linear chirp. IET Radar, Sonar and Navigation, 11(1):142-153, 2017.

[25] Aleksandar Dogandžić and Arye Nehorai. Cramér-Rao bounds for estimating range, velocity, and direction with an active array. IEEE Transactions on Signal Processing, 49(6):1122-1137, jun 2001.

[26] Steven M Kay 1951-. Fundamentals of statistical signal processing estimation theory / Steven M. Kay. PTR Prentice Hall, c1993., 1993.

[27] Stephen J Wright. Coordinate Descent Algorithms. pages 1-32. 